\title{
Métodos para la detección oportuna de cáncer cervicouterino
}

\author{
Methods for Cervical Cancer Screening
}

\author{
Tatiana Vargas-Revilla ${ }^{1}$ \\ Jesús Manuel Seáñez-de-Villa² \\ Noel León-Rovira ${ }^{3}$ \\ Olivia Maricela Barrón-Cano ${ }^{4}$
}

Fecha de recepción: 14 de abril de 2014

Fecha de aceptación: 28 de julio de 2014

Vargas-Revilla, T; Seáñez-de-Villa, J; León-Rovira, N;

Barrón-Cano, O. Métodos para la detección oportuna

de cáncer cervicouterino. Tecnología en Marcha.

Edición especial Movilidad Estudiantil 20 I4. Pág. 52-59.

Estudiante de Ingeniería en Producción Industrial del Instituto Tecnológico de Costa Rica. Correo electrónico: tatisvr@gmail.com

2 Soluciones en Dispositivos Médicos S de RL de CV, Ave. Eugenio Garza Sada \#427 Sur, Pabellón Tec, Local 38 interior Medicí, Col. Linda Vista, C.P. 64849, Monterrey, Nuevo León, México.

3 Centro de Innovación en Diseño y Tecnología, Escuela de Ingeniería y Tecnologías de Información, Instituto Tecnológico y de Estudios Superiores de Monterrey, CETEC, 5to piso, Torre Norte, Ave. Eugenio Garza Sada 250 I, Monterrey, Nuevo León, México.

4 Soluciones en Dispositivos Médicos S de RL de CV, Ave. Eugenio Garza Sada \#427 Sur, Pabellón Tec, Local 38 interior Medicí, Col. Linda Vista, C.P. 64849, Monterrey, Nuevo León, México. 


\section{Palabras clave}

Cáncer cervicouterino; sensibilidad; especificidad.

\section{Resumen}

El cáncer cervicouterino afecta a gran parte de la población femenina a nivel mundial, en países subdesarrollados como Costa Rica llegó a ser la tercera causa de muerte. El método más común para su detección es el Papanicolau; sin embargo, presenta niveles de sensibilidad y especificidad muy elevados, por lo que diferentes entidades han desarrollado diversos métodos para su detección. El artículo comprende tres secciones: la primera abarcará el impacto que ha tenido el cáncer cervicouterino en Costa Rica, en términos generales; la segunda, una recopilación de diferentes metodologías para la detección del cáncer alrededor del mundo; y la tercera, el desarrollo actual de un dispositivo en México que actúa como un sistema de monitoreo y su diseño permite ser utilizado por la misma mujer sin asistencia externa.

\section{Key words}

Cervical Cancer; sensitivity; specificity.

\section{Introducción}

El cáncer cervicouterino se refiere al cáncer de cérvix, situada en la parte baja del útero, una de las causas principales de muerte en las mujeres (Alvarado González, 20।2). El cáncer cervicouterino es un problema de salud pública en la mayoría de los países del mundo y, principalmente, en las naciones en vías de desarrollo ya que el $80 \%$ de las muertes por esa causa ocurren en países pobres. En Latinoamérica y el Caribe la incidencia llega al doble, y la mortalidad al triple de la que se presenta en los países desarrollados (Morera Salas \& Aparicio Llanos, 2007).

Costa Rica no cuenta con estudios recientes acerca de índices de mortalidad a causa de este cáncer; sin embargo, constituyeron la tercera causa de muerte por cáncer en las mujeres (tasa de 8.6/l 00 mil), en el período 2000-2005. La mitad de estas defunciones se produjeron entre los 15 y 64 años, consideradas como muertes evitables por existir intervenciones suficientemente efectivas para prevenirlo(Aparicio Llanos \& Morera Salas, 2007).

\begin{abstract}
Cervical cancer affects a great portion of the world's female population,and it became the third cause of death for women in developing countries such as CostaRica.The most common method to diagnose this cancer is the Papanicolaou testor Papsmear; nevertheless, high levels of sensitivity and specificity are required. Consequently, different organizations have developed multiple methods to detect and classify this cancer.

This article is divided in three sections: the first one focuses on the general impact of cervical cancer has hadin CostaRica, these condsection gathers information about different methodologies used around the world to detect this cancer and the third one makes reference to the current development of the screening devise in Mexico that works as a monitoring system and can used by women without external assistance.
\end{abstract}

Por otro lado, un estudio realizado por el Ministerio de Salud Pública de Costa Rica revela que las tasas de incidencia han variado considerablemente en los periodos 2000-20l0. Entre el 2003 y el 2008, se presenta una tendencia descendente (tasa de 48.64 a una tasas de 26.55), la cual se invierte a partir de este año, que pasó de una tasa de 26.55, a una tasa de 32.5I por cada 100.000 mujeres en el 2010 (Ministerio de Salud de Costa Rica, 2013).

El cáncer cervicouterino se incluye en el grupo de causas tratables donde las muertes pueden ser evitadas por un diagnóstico temprano y un tratamiento oportuno(Morera Salas \& Aparicio Llanos, 2007). El método tradicional para la detección del cáncer cervocouterino es el Papanicolau o citología cervical, consiste en extraer una muestra del tejido de la parte baja del útero, el cérvix, mediante el uso de un cepillo o "citobrush" para, luego, ser examinada en un laboratorio, generalmente, con ayuda de un patólogo. Este método presenta varias desventajas entre las que destacan el alto índice de 
falsos diagnósticos negativos, los altos costos de laboratorio y el mismo procedimiento que presenta sus propios retos que van desde la incomodidad de la mujer, el tiempo de espera para obtener los resultados, hasta factores sociales como el machismo y la ignorancia o falta de cultura de prevención.

Esta investigación tiene por objetivo dar a conocer parte de las nuevas tecnologías existentes para la detección del cáncer cervicouterino mediante un cuadro comparativo de las principales características. Y más específicamente, el desarrollo de un dispositivo móvil para la detección de cáncer cervicouterino que se encuentra en proceso de desarrollo en el Tecnológico de Monterrey, Campus Monterrey, con ayuda de un Spin Off llamado Soluciones en Dispositivos Médicos S de RL de CV, una nueva opción, menos invasiva, fácil de usar y que da un resultado en tiempo real.

\section{Metodologías para la detección de cáncer cervicouterino}

En los últimos años, varias compañías se han dedicado a desarrollar dispositivos médicos que faciliten la detección temprana de cánceres, y el caso del cáncer cervicouterino no es la excepción. Este impulso de crear nuevas tecnologías ha surgido por la necesidad de aumentar los niveles de confiabilidad en los resultados, reducir los costos de exámenes y disminuir los tiempos de respuestas.

En la figura I, se muestra el diagrama general sobre el proceso o protocolo seguido para la detección y tratamiento de este cáncer. Se considera un resultado positivo desde que se arroja un resultado CINI y CIN2 (lesiones intraepiteliales escamosas de bajo grado); en estas etapas solo requiere control y seguimiento, y cuando hay CIN3 o displasia in situ (lesiones de alto grado y principio de cáncer) es dondese debe aplicar tratamiento.

Para desarrollar el cuadro recopilatorio de las metodologíasque se han aplicado alrededor del mundo, se seleccionaron6 parámetros como punto de comparación. Los parámetros son sensibilidad, especificidad, toma de muestra de tejido, intervención técnica en la interpretación de resultados, tiempo de respuesta y si el equipo es portátil. Primeramente, es importante definir que es sensibilidad y especificidad, según Pita Fernández, S y Pértegas Díaz, S(20 I0) en su artículo sobre pruebas diagnósticas:

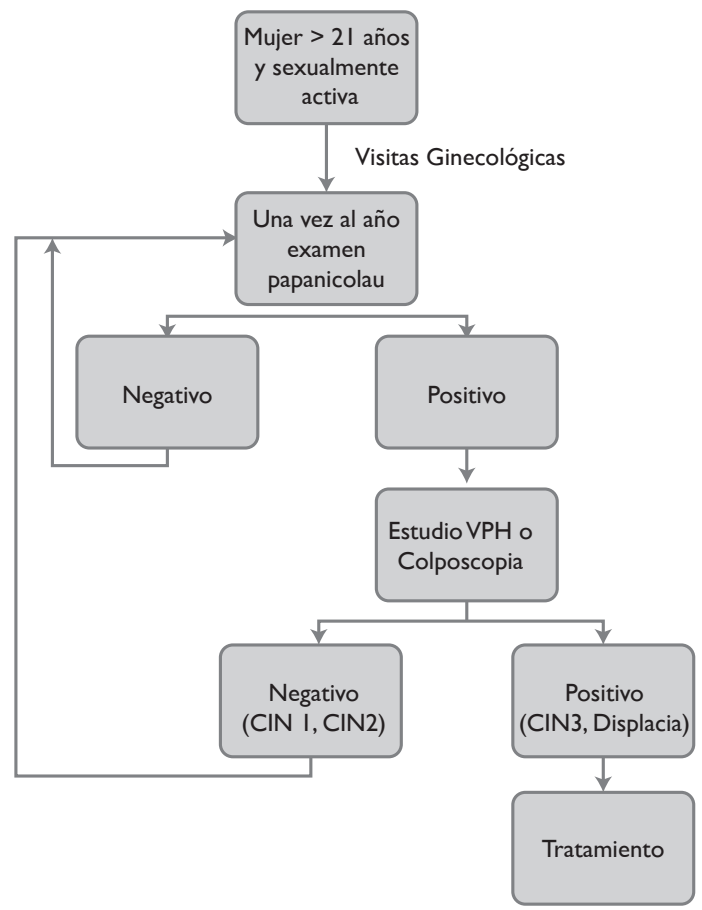

Figura I. Diagrama de seguimiento de salud cervical.

Sensibilidad: es la probabilidad de clasificar correctamente a un individuo enfermo, es decir, la probabilidad de que para un sujeto enfermo se obtenga un resultado positivo. La sensibilidad es, por tanto, la capacidad del test para detectar la enfermedad.

Especificidad: es la probabilidad de clasificar correctamente a un individuo sano, es decir, la probabilidad de que para un sujeto sano se obtenga un resultado negativo. En otras palabras, se puede definir la especificidad como la capacidad para detectar a los sanos.

En el Cuadro I se muestra 13 metodologías que se han desarrollado en los últimos 20 años, algunas comparten principios de funcionamiento que se explicaran a continuación.

El Papanicolau es el método más usado para la detección de cáncer cervicouterino; sin embargo, no es una prueba de diagnóstico fehaciente por sí sola, sino que se combina con diferentes tecnologías o métodos para determinar la presencia de cáncer - precáncer. Comúnmente se realiza la prueba de Colposcopia después de encontrar células anormales, esta prueba consiste en utilizar un colposcopio para examinar el cuello uterino de manera que el doctor 
Cuadro I. Tabla resumen sobre las metodologías para la detección de cáncer cervicouterino

\begin{tabular}{|c|c|c|c|c|c|c|}
\hline Dispositivo & $\begin{array}{l}\text { Toma de } \\
\text { Muestra de } \\
\text { tejido }\end{array}$ & $\begin{array}{l}\text { Intervención técnica } \\
\text { en la interpretación } \\
\text { de resultados }\end{array}$ & $\begin{array}{c}\text { Especificidad } \\
\text { (falsospositivos) }\end{array}$ & $\begin{array}{c}\text { Sensibilidad } \\
\text { (falsosnegativos) }\end{array}$ & $\begin{array}{l}\text { Tiempo de } \\
\text { respuesta } \\
\text { inmediata }\end{array}$ & $\begin{array}{l}\text { Equipo } \\
\text { Portátil }\end{array}$ \\
\hline $\begin{array}{l}\text { Papanicolaou } \\
\text { (método } \\
\text { tradicional) }\end{array}$ & Sí & Sí & $72 \%[1]$ & $84.1 \%[1]$ & No & No \\
\hline Colposcopia & No & Sí & $\begin{array}{l}45 \%[1] \\
47 \%[2] \\
25 \%[3]\end{array}$ & $\begin{array}{r}>88.1[1] \\
78 \%[2] \\
79 \%[3]\end{array}$ & Sí & No \\
\hline ADN de VPH & Sí & Sí & $44 \%[4]$ & $88 \%[4]$ & No & No \\
\hline $\begin{array}{l}\text { ThinPrep } \\
\text { (De base líquida) }\end{array}$ & $S$ & Sí & $97.8 \%[5]$ & $82 \%[5]$ & No & No \\
\hline Pathfinder & Sí & Sí & - & - & - & No \\
\hline Papnet & Sí & Sí & $99.3 \%[6]$ & $97.10 \%[6]$ & No & No \\
\hline TruScreen & No & Sí & $92.4 \%[7]$ & $85.1 \%[7]$ & Sí & No \\
\hline $\begin{array}{l}\text { AutoPap } 300 \\
\text { QC System * }\end{array}$ & Sí & Sí & - & - & No & No \\
\hline $\begin{array}{l}\text { LuViva® } \\
\text { Advanced } \\
\text { Cervical Scan }\end{array}$ & No & No & $84-88 \%$ [8] & $50-60 \%[8]$ & Sí & No \\
\hline ZedScan & No & No & $65.1 \%[9]$ & $89 \%[9]$ & Sí & No \\
\hline DySIS & No & No & $62.60 \%[10]$ & $79.6 \%[10]$ & Sí & No \\
\hline Niris & No & No & $81.10 \%[10]$ & $87.20 \%[10]$ & Sí & No \\
\hline $\begin{array}{l}\text { Propuesto } \\
\text { (CACU) }\end{array}$ & No & No & $>85 \%$ & $>85 \%$ & Sí & Sí \\
\hline
\end{tabular}

* Este producto ya no es comercial

- No hay información en la web sobre esa rúbrica

[1] Rojas Mendoza, G., Córdoba Uscanga, C., \& Sanchez López, J. (20l2). Evaluación del estudio de Papanicolar y la coloscopia en el diagnóstico de neoplasia intraepitelial cervical en la Unidad Especial Centro de Apoyo Diagnóstico San Rafael. Revista de Especialidades Médico-Quirurgicas, 76-80.

[2]Uzcátegui,Y.Tovar, M. Lorenzo C., \& González, M. (20 I2). Patología vaginal: utilidad de la citología y la colposcopia como métodos diagnósticos. Revista Obstetricia Ginecológica, 161-170.

[3] Cabrera, J (2003). Evaluación de la Citología Cervicovaginal y la Colposcopia, como Método de Detección de la Neoplasia Intraepitelial Cervical. Rev. Per Ginecol Obstet. I55- 159

[4] Isaza Ruget, M. A., Pérez, G., Morales Reyer, O., Deantonio Suárez, R., Alvarado Heine, C., \& Trujillo, L. M. (2009). Exactitud del test ADN-HPV para la detección de la enfermedad cervical de alto grado (NIZ 2+) en mujeres con anormalidades citológicas (ASC-US y LSIL), afiliadas a la seguridad social en Bogotá . Revista Colombiana de Obstetricia y Ginecología , 60(3), 21 3-222.

[5] Biscotti CV, D., Dziura B, G. L., \& Darragh T, R. (2005). Assisted primary sreening using the automated ThinPrep Imaging System. American journal of clinical pathology, |23-29|.

[6] Internacional Agency for Research on Cancer. (2005). Cervix Cancer Screening . IARC Press, I0, 59- I I 5.

[7] Sung Jong Lee, Jeong Hoon Bae, Jin Hwi Kim, Sung Ha Lee, Sung Eun Namkoong, \& Jong Sup Park. (2009). A real-time optoelectronic device in screening of cervical intraepithelial neoplasia. Journal of Women's Medicine, 2(I), 23-28.

[8] Flowers, L., Ault, K., Twiggs, L., Chakhtoura, N.,Wilkinson, E., Ferris, D., ... Sternfeld, D. (20 I2). Guided Therapeutics. Recuperado el II de I0 de 20 I3, de http://www.guidedinc.com/Pub\%20data/ASCCP\%202012\%20Poster.pdf

[9] ZedScan. (20/3). Zilico. Obtenido de http://www.zilico.co.uk/products/clinical-studies-results.html

[10] R Wade, E Spackman, M Corbett, S Walker, K Light, R Naik, M Sculpher\&A Eastwood (2013). Adjunctive colposcopy technologies for examination of the uterine cervix - Dysls, luViva advanced cervical scan and nirislmaging system: a systematic review and economic evaluation. HealthTechnologyAssessment. 17(8), 14-22 
observe la superficie del cuello del útero y detecte anormalidades para luego descartar el cáncer, o bien proceder a una biopsia.

Los valores de sensibilidad y especificidad para el Papanicolau no son los reportados en Costa Rica ya que no se cuenta con estudios sobre estos índices. Sin embargo, los valores mostrados están en el rango de acuerdo con varios estudios; el índice de sensibilidad no es tan alto como se esperaría y por eso se han creado nuevas metodologías para reafirmar los resultados, como es el uso de la colposcopia que puede llegar a tener un índice de sensibilidad, por lo menos, $4 \%$ mayor; también se puede observar cómo en el 2003 los índices para Colposcopia eran mucho menores a los obtenidos recientemente (considerando que existen en la literatura bastantes artículos que analizan estos números sobre los cuales se pueden encontrar grandes variaciones).

El uso de imágenes computarizadas e inteligencia artificial es el principio de funcionamiento para la detección del cáncer en metodologías como AutoPapSystem y PapNet. En un artículo del Centro Internacional de Investigación de Cánceres menciona que la diferencia entre estas metodologías se debe a que el AutoPap, revalúa los Pap negativos y selecciona la población de mayor riesgo para anomalías. El PapNet fue diseñado como complemento del sistema manual de la recolección de la muestra y selecciona las 128 imágenes anormales para su posterior revisión.PapNet es una metodología con índices de sensibilidad y especificidad bastante altos, no obstante el uso de esta tecnología implica un aumento considerable en el costo del examen.

Por otro lado, la mejora en los procesos de tamizaje también son principios que se han ido mejorando y aplicando en las nuevas metodologías. ThinPreplmagingSystem es la mezcla de imágenes computarizadas y mejora en la prueba de tamizaje, el método consiste en extraer la muestra del paciente de la misma manera que el Papanicolau, pero se aclara inmediatamente en un vial de solución conservante (base líquida). La muestra se envía al laboratorio, donde se procesa utilizando un instrumento automatizado que elimina parte de la muestra que no sirve y transfiere una muestra representativa de las células en una capa delgada en la diapositiva para la prueba de PapThinPrep.

Metodologías como la prueba de ADN de VPH consisten en realizar la prueba de Papanicolau junto con la prueba de VPH. La infección del virus del papiloma humano (VPH) es reconocida como causa del cáncer cervical (Valderrama, Campos, Cárcamo, \& García, 2007), de manera que esta prueba identifica el virus que puede estar causando la aparición de células anormales en el cuello del útero. Esta prueba tiene mayor grado de sensibilidad que el Papanicolau ya que son complementos; sin embargo, tiene un bajo grado de especificidad.

También existen las metodologías que utilizan la optoelectrónica para detectar la presencia del cáncer como es el caso de TruScreen. TruScreen es un dispositivo opto-electrónico, que utiliza señales eléctricas y ópticas de bajo nivelpara escanear el cuello del útero. La respuesta se mide y el software del dispositivo basado en computadora clasifica la respuesta de los tejidos; por medio de la comparación de las señales almacenadas su base de datos con la gama de tipos de tejidos del cuello del útero (Pruski, y otros, 2008) Este tipo de tecnología permite dar una respuesta inmediata.

Otras metodologías son aquellas que funcionan como complemento de las Colposcopias como son LuViva, NirislmagingSystem, DySis y ZedScan. LuViva es un sistema de análisis digital de imágenes para la detección de tejido cervical canceroso y precanceroso. Funciona mediante la detección de cambios bioquímicos y morfológicos en el nivel celular (utilizando espectroscopia óptica). (Wade, y otros, 2013), señalan queestos métodos tienen las siguientes características:

NirislmagingSystemes otro sistema de análisis digital de imágenes para la detección de tejido cervical canceroso y precanceroso. Funciona utilizando tomografía de coherencia óptica para producir una imagen de dos dimensiones del tejido.

DYSIS Es un colposcopio que incorpora una cámara digital utilizando imágenes espectrales dinámicas para la detección de tejido cervical canceroso y precanceroso. Funciona, después de la aplicación de ácido acético, mediante la asignación del blanqueado con ácido acético del epitelio del cuello uterino (la DySISmap).

De las metodologías basadas en imágenes, LuViva es la que tiene el menor índice de sensibilidad y a la vez tiene mayor índice de especificidad, en este tipo de pruebas es preferible centrarse en los valores de sensibilidad donde Niris tiene el valor más alto. 
ZedScanutiliza Impedancia Eléctrica (EIS) para identificar y diferenciar los tipos de tejido del cuello del útero en función de sus propiedades eléctricas. Estas propiedades son conocidas al cambiar durante el desarrollo del cáncer, con más células displásicas muestran una disminución de la resistencia al flujo de corriente como resultado de los cambios estructurales, en otras palabras, mientras más avanzado se esté en el desarrollo del cáncer menor es la resistencia que opone el tejido al paso de corriente (ZedScan, 20 I3).

Proyecto Cacues un dispositivo que se encuentra en desarrollo en el Tecnológico de Monterrey con apoyo de un "Spin Off" conocido como Soluciones en Dispositivos Médicos S de RL de CV, se busca desarrollar un método de monitoreo para un pre-diagnóstico del cáncer. El funcionamiento del instrumento está basado en mediciones ópticas y electrónicas. En la siguiente sección se explicará con más detalle.

\section{Descripción del Proyecto $\mathrm{CaCu}$}

El Proyecto de Detección de Cáncer Cérvico Uterino (Proyecto CACU) inició en el 2003, con la idea del Dr. Luis A. Perez Romeroen el Tecnológico de Monterrey, el objetivo era diseñar un dispositivo práctico y portátil para que se pudiera obtener una muestra de cérvix de manera rápida y efectiva. El objetivo fue evolucionando hasta su versión actual que contempla el proporcionar un sistema de monitoreo, que inclusive pueda ser utilizado por la misma mujer sin asistencia externa.

Actualmente, el dispositivo se encuentra en la etapa de prototipo y cuenta con dos patentes otorgadas en México, MX 308829 B. "Dispositivo personal para toma de muestras de células y virus del endocérvixy exocérvix". Y MX26|228. "Dispositivo portátil para prueba de autodiagnóstico de cáncer cérvico uterino por medio de mediciones eléctricas y ópticas simultáneas". Además, de otra patente publicada MX/a/2008/0I5279 titulada "Dispositivo portátil mejorado para prueba de autodiagnóstico de cáncer cérvico uterino por medio de mediciones eléctricas y ópticas simultáneas" y una última publicada a nivel internacional PCT/MX2006/0000 I I "Optoelectronicdeviceforthedetection of uterine cervical cancer, comprising a selfpositioningattachment".

En la figura 2, se explica brevemente el funcionamiento interno del dispositivo.

La unidad de mediciones ópticas se encarga de enviar señales lumínicas de color rojo, verde y

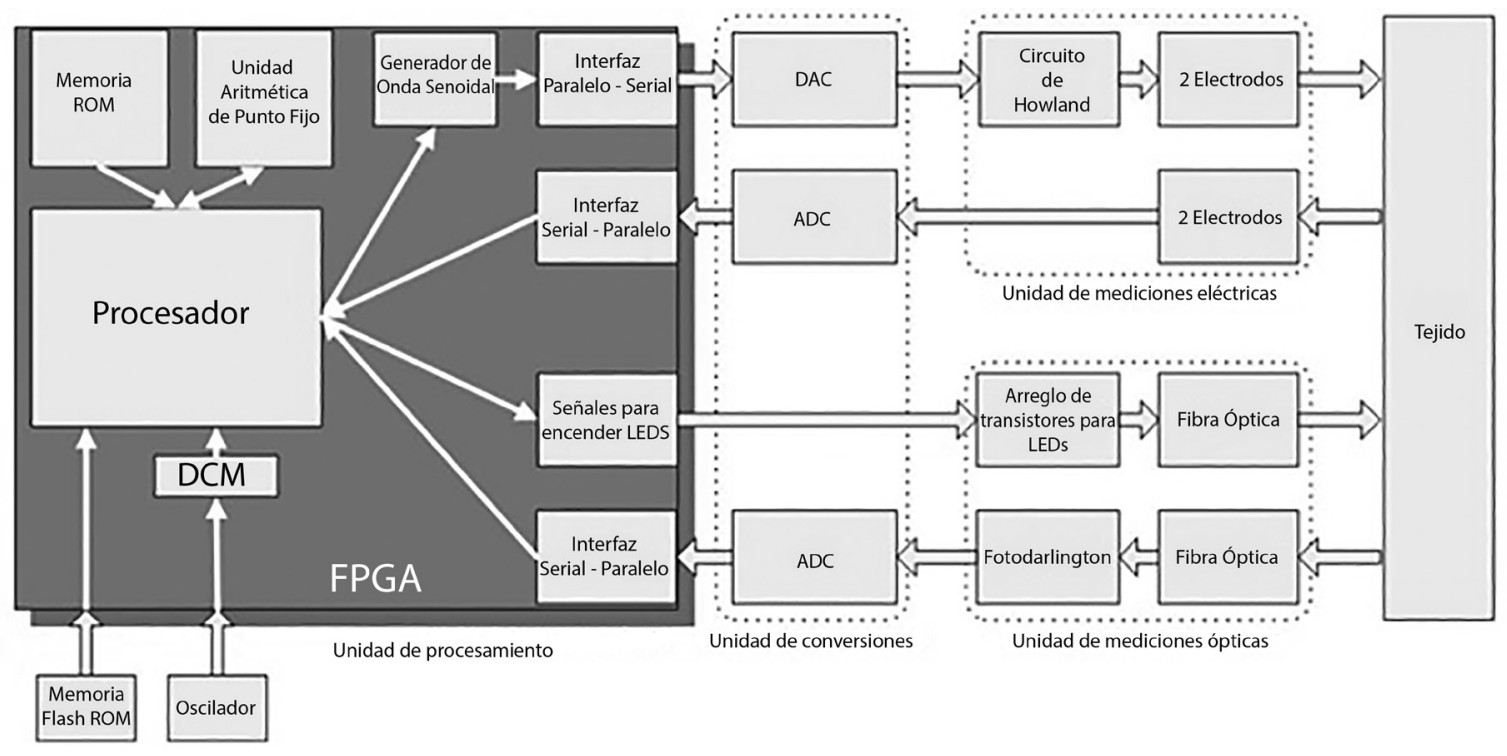

Fuente: (Seañez \& Aguilar Alemán, 2007)

Figura 2. Diagrama de bloques funcional $\mathrm{CaCu}$ 
azul por medio de LEDs al tejido y de medir sus características de refracción de luz a través de un fotodarlington conectado a una resistencia, que convierte la señal de luz en una señal eléctrica. La unidad de mediciones eléctricas está basada en el circuito de Howland, ideal para dispositivos que utilicen como fuente de poder baterías.

La unidad de conversiones se basa en dos convertidores, uno análogo-digital para las mediciones eléctricas y ópticas y otro convertidor digital análogo para la generación de la onda senoidal de entrada a la unidad de medición óptica.

La unidad de procesamiento se encarga de procesar la información proveniente de los convertidores, a través de ella se generan el resultado final de la prueba desplegando diferentes colores a través de los LED, color rojo para tejido con cáncer, amarillo para lesiones de bajo y alto grado, y verde para el tejido sano.

El dispositivo ha pasado por varias etapas de diseño debido a ajustes en la parte electrónica del dispositivo, material en la parte interna y externa del instrumento, ensamble de las diferentes partes, dimensiones de la tarjeta, entre otros, para concluir con el prototipo de la figura 3.

Actualmente, el dispositivo se encuentra en etapa de prototipo beta y los protocolos de pruebas clínicas en humanos están en trámite de aceptación ante los organismos regulatorios en México. Dichas pruebas permitirán al equipo de desarrollo establecer mediante evidencia supervisada los niveles de confiabilidad (sensibilidad y especificidad) de la tecnología; sin embargo, los resultados preliminares han permitido asegurar niveles de ambos parámetros mayores al $85 \%$. Por esto, y por los resultados por obtener, que en el cuadro $\mathrm{NI}$ en ambas columnas se indica $>85 \%$ (mayor al $85 \%$ ) en espera de fijar estos valores al finalizar las pruebas en humanos.

\section{Conclusión}

En Costa Rica los métodos para la detección de Cáncer Cervicouterinoutilizados en los Servicios Públicos de Salud son el Papanicolau y la Colsposcopia; sin embargo, no existen estudios recientes sobre sus niveles de especificidad y sensibilidad. Teniendo estos datos, se podrían tomar acciones correctivas de manera que no se encuentre dentro de las primeras

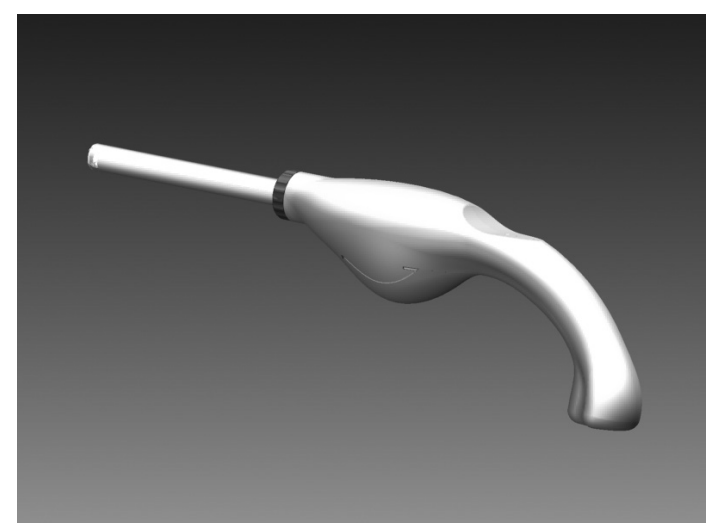

Figura 3. Prototipo final $\mathrm{CaCu}$.

causas de muerte en la población femenina ya que es una enfermedad totalmente tratable en las etapas iniciales.

De acuerdo con nuestra revisión, una de las causas principales por la que no se detecta a tiempo el cáncer cervicouterinoes de carácter social; es decir, existe un tabú acerca del examen de Papanicolau y Colposcopia que hace percibir el procedimiento como doloroso, aunado con timidez de realizarse el examen y el costo que implica hacerlo. No obstante, existen gran cantidad de métodos como los mencionados en el cuadro $\mathrm{NI}$ que no son invasivos y ofrecen resultados confiables; sin embargo, no se han llegado a implementar en la salud pública del país.

El desarrollo del proyecto $\mathrm{CaCu}$ facilitaría a la población femenina realizarse una detección temprana del cáncer, hasta en una etapa de precáncer, con el fin de disminuir los índices de mortalidad a causa de esta enfermedad. El dispositivo, además de ser un autoexamen, ofrecería un tiempo inmediato de respuesta, con un costo accesible para todas las clases sociales y con un alto grado de confiabilidad.

La etapa en la que se encuentra actualmente (protocolo) y las que hacen falta para concluir el dispositivo, se visualiza dos obstáculos que se pueden dar a nivel social y de diseño. Las pruebas médicas se pueden ver afectadas por fallos en el diseño; para evitar estos fallos es necesario cumplir con protocolos que permitan ir realizando pruebas en el funcionamiento como en la tarjeta electrónica y de calibración. En caso de que no se lleve a cabo puede afectar en el tiempode salida al mercado e impacto económico. 
A nivel social, se debe de realizar una introducción gradual en el mercado, primero el médico,y luego la paciente realizándose el autoexamen. En esta última etapa se debe tener en cuenta que si la paciente no cumple con las normas de uso la prueba puede llegar a no ser certera; además, se debe educar a las mujeres sobre cómo realizarlo correctamente y que no tengan miedo.

\section{Agradecimientos}

Los autores expresan su agradecimiento al "Programa de Pasantía Estudiantil con Fondos del Sistema 2014, CONARE-TEC" con el cual se ha cubierto parte fundamental de los gastos de viaje del pasante y a la Rectoría del Instituto Tecnológico que gestiona todo el programa.

\section{Bibliografía}

Alvarado González, C. (20 I2). Metodología de diseño y manufactura de productos en empresas pequeñas. Monterrey, Nuevo León, México.

Aparicio Llanos, A., \& Morera Salas, M. (2007). Variabilidad geográfica en la detección temprana del cáncer cervicouterino entre servicios integrados de atención en Costa Rica, 2004 - 2005. Revista Costarricense de la Salud Pública, 54-6l.

Biscotti CV, D., Dziura B, G. L., \& Darragh T, R. (2005). Assisted primary sreening using the automated ThinPrep Imaging System. American journal of clinical pathology, 123-291.

Díaz Pértegas, S., \& Pita Fernández, S. (20 l0). Fisterra. Obtenido de Pruebas Diagnósticas: Sensibilidad y Especificidad. Recuperado desde: https://www.fisterra.com/mbe/investiga/ pruebas_diagnosticas/pruebas_diagnosticas.asp

Flowers, L., Ault, K., Twiggs, L., Chakhtoura, N., Wilkinson, E., Ferris, D.,... Sternfeld, D. (20।2). Guided Therapeutics. Recuperado desde: http://www.guidedinc.com/Pub\%20data/ASCCP\%20 2012\%20Poster.pdf

Internacional Agency for Research on Cancer. (2005). Cervix Cancer Screening . IARC Press, 10, 59-1 I 5.

Isaza Ruget, M.A., Pérez, G., Morales Reyer, O., Deantonio Suárez, R., Alvarado Heine, C., \& Trujillo, L. M. (2009). Exactitud del test ADN-HPV para la detección de la enfermedad cervical de alto grado (NIZ 2+) en mujeres con anormalidades citológicas (ASC-US y LSIL), afiliadas a la seguridad social en Bogotá . Revista Colombiana de Obstetricia y Ginecología, 60(3), 213-222.

Ministerio de Salud de Costa Rica. (20।3). Memoria Institucional. San José: El Ministerio.

Morera Salas, M., \& Aparicio Llanos, A. (2007). Análisis geográfico y temporal de la mortalidad evitable por cáncer cervicouterino en Costa Rica. Acta Médica Costarricense, |54- 160.

National Institute for Health and Care Excellence. (Agosto de 2012). NICE. Recuperado desde: http://www.nice.org.uk/ nicemedia/live/ I 3842/60280/60280.pdf

Pruski, D., Kedzia, W., Przybylski, M., Kedzia, H., Jozefiak, A., \& Spaczynski, M. (2008). The evaluation of a real-time optoelectronic method for the detection of Cervical Intrepithelial Neoplasia ("CIN'), I-6.

Rojas Mendoza, G., Córdoba Uscanga, C., \& Sanchez López, J. (20।2). Evaluación del estudio de Papanicolar y la coloscopia en el diagnóstico de neoplasia intraepitelial cervical en la Unidad Especial Centro de Apoyo Diagnóstico San Rafael. Revista de Especialidades Médico-Quirurgicas, 76-80.

Seañez, J., \& Aguilar Alemán, J. (2007). Towards reliable cancer diagnosis using incomplete and/or noisy data. Dispositivo Portátil de Diagóstico de CaCu. Monterrey, Nuevo León, México.

Sung Jong Lee, Jeong Hoon Bae, Jin Hwi Kim, Sung Ha Lee, Sung Eun Namkoong, \& Jong Sup Park. (2009). A real-time optoelectronic device in screening of cervical intraepithelial neoplasia. Journal of Women's Medicine, 2(I), 23-28.

Valderrama, M., Campos, F., Cárcamo, C., \& García, P. (2007). Factores asociados a lesiones cervicales o presencia del virus del papiloma humano en dos poblaciones de estudiantes de Lima. Revista Peruana de Medicina Experimental y Salud Publica, 235-239.

Wade, R., Spackman, E., Corbett, M., Walker, S., Light, K., Naik, R., ... Eastwood, A. (20/3). adjunctive colposcopy technologies for examination of the uterine cervix - Dysls, luViva advanced cervical scan and niris Imaging system: a systematic review and economic evaluation. Health Technology Assessment, |-17|.

ZedScan. (20|3). Zilico. Recueprado desde: http://www.zilico. co.uk/products/clinical-studies-results.html 